\title{
Disparities in Postpartum Contraceptive Counseling and Provision Among Mothers of Preterm Infants
}

\author{
Annie Dude, MD, PhD, , Melissa Matulich, MD, Samantha Estevez, BA, \\ Lilly Y. Liu, MD, MPH, and Lynn M. Yee, MD, MPH ${ }^{1}$
}

\begin{abstract}
Objective: Use of effective contraception could be one method to decrease recurrent preterm birth by increasing intervals between pregnancies. We assessed correlates of contraceptive counseling and uptake among women who delivered preterm.

Materials and Methods: This is a retrospective cohort study of women who delivered live-born singletons or twins before 32 weeks' gestation. We assessed documented contraceptive counseling and method uptake by postpartum discharge, using inpatient medical records, and correlates of highly effective contraception uptake by the postpartum visit using outpatient records.

Results: Of 594 eligible women, 44.6\% $(n=265)$ had documented inpatient contraceptive counseling, but only $21.4 \%(n=127)$ left the hospital using a World Health Organization (WHO) tier 1 or 2 method. Women who were younger, non-Hispanic black, multiparous, and delivered at earlier gestational ages were more likely to have inpatient counseling documented. Compared with women with private insurance, women with public insurance were more likely to have documented counseling $(22.8 \%$ vs. $87.5 \%, p<0.001$; adjusted odds ratio [aOR] 9.55, 95\% confidence interval [CI $5.31-17.2)$ and to uptake a WHO tier 1 or 2 method as an inpatient (5.8\% vs. 52.0\%, $p<0.001$; aOR 9.51, 95\% CI 4.78-18.9). Of the 175 women with outpatient records available who attended a postpartum visit, only $54.9 \%(n=96)$ adopted a WHO tier 1 or tier 2 method.

Conclusion: Although all women in this cohort were at risk of recurrent preterm birth, counseling about contraception after a preterm birth ( $<32$ weeks) was not universal. Women with multiple risk factors for recurrent preterm birth, such as multiparity and public insurance, were more likely to have received documented contraceptive counseling and highly effective contraceptives.
\end{abstract}

Keywords: contraception, health disparities, postpartum

\section{Introduction}

$\mathbf{P}$

RETERM BIRTH OCCURRED in $9.6 \%$ of all live births in the United States in $2015^{1}$ and is a significant cause of neonatal mortality ${ }^{2}$ and child morbidity. ${ }^{3,4}$ Moreover, preterm birth is a reproductive health disparity as racial and ethnic minority women are disproportionately at risk of preterm birth and subsequent sequelae, including neonatal demise, cerebral palsy, and long-term neurocognitive deficits. ${ }^{1,5}$ Thus, prevention of preterm birth remains of utmost clinical and public health importance. ${ }^{6}$
Although the etiology of preterm birth is poorly understood, major risk factors for preterm birth include prior preterm delivery ${ }^{7}$ and a short interpregnancy interval. ${ }^{8} \mathrm{~A}$ short interpregnancy interval (often defined as $\leq 18$ months between delivery of one pregnancy and conception of the next) is associated with increased risk of index or recurrent preterm birth independent of other confounding factors ${ }^{9}$ and is associated with increased neonatal morbidity at any gestational age. ${ }^{10}$

Approximately one-third of pregnancies in the United States are conceived following a short pregnancy interval. ${ }^{11}$ Although the association between interpregnancy interval

\footnotetext{
${ }^{1}$ Division of Maternal-Fetal Medicine, Department of Obstetrics and Gynecology, Feinberg School of Medicine, Northwestern University, Chicago, Illinois.

${ }^{2}$ Department of Obstetrics and Gynecology, Feinberg School of Medicine, Northwestern University, Chicago, Illinois

${ }^{3}$ Feinberg School of Medicine, Northwestern University, Chicago, Illinois.

${ }^{4}$ Department of Obstetrics, Gynecology and Reproductive Science, Icahn School of Medicine at Mount Sinai, New York, New York.

This abstract was presented at the Society for Maternal-Fetal Medicine Annual Meeting, January 2017, Las Vegas, NV (Abstract \#389).
} 
and preterm birth remains under investigation, ${ }^{12,13}$ short intervals are more common among women already at higher risk of preterm birth: women who are non-Hispanic black or Hispanic, young, unmarried, less educated, and with higher parity. ${ }^{14}$ This phenomenon is often due to racial or ethnic and socioeconomic disparities in access to contraception. ${ }^{15-17}$

Much focus on prevention of recurrent preterm birth is on management during a subsequent pregnancy. However, spacing a subsequent pregnancy via effective contraceptive use is potentially an under-recognized preterm birth prevention strategy. ${ }^{18}$ Antenatal and postpartum contraceptive counseling can improve the uptake and use of effective contraception. ${ }^{19}$ In this study, we aimed to identify disparities in and correlates of contraceptive counseling and uptake among women who delivered before 32 weeks' gestational age, a population at high risk of recurrent preterm birth. ${ }^{7}$

\section{Materials and Methods}

This is a retrospective cohort study of women who delivered live-born singleton or twin gestations between 23 and 32 weeks' gestation at a single tertiary care institution from January 1, 2011, to June 30, 2015. We included twin gestations as well as singletons as previous research indicates that women with a previous twin delivery remain at risk of preterm delivery in a subsequent singleton pregnancy. ${ }^{20}$ This academic tertiary care institution serves a diverse population of privately and publicly insured pregnant women and is a referral hospital for the local perinatal network.

At this institution, all women hospitalized antenatally are seen by both resident and attending physicians daily. Residents are involved in the intrapartum care of all women experiencing preterm birth. Following delivery, however, arrangements for postpartum rounding vary. Both resident and attending physicians care for women who had no prenatal care, received care in an affiliated federally qualified health center network, were transferred in from outside hospitals, and who received care in the resident-run ambulatory clinics at this institution and all mothers with any postpartum complication (such as sepsis, endometritis, hypertension, or hemorrhage).

Women with academic faculty or private practice providers whose pregnancies and postpartum courses are otherwise uncomplicated are only seen by an attending physician, advanced practice nurse, or certified nurse midwife, as per individual practice policies. All resident physicians at this institution are trained to provide a full range of contraceptives, including intrauterine devices (IUDs) and subdermal implants, although these devices were only available in the outpatient setting during the study period.

Data were abstracted from inpatient electronic medical records, including provider notes, procedure notes, and medication reconciliation records. For all women, the inpatient chart was reviewed to identify whether they were counseled by a provider (physician, certified nurse midwife, or advanced practice nurse) regarding postpartum contraception and to determine a planned or actual contraceptive method at the time of hospital discharge following delivery. All available antepartum and postpartum inpatient notes were examined and women were determined to have been counseled if any of these notes contained any documentation of discussion of contraceptive choice (even if the patient ultimately did not select a method).
Outpatient records were additionally available for a subset of women receiving postpartum care with a provider who uses the university-based electronic medical record, a subset that included both publicly insured women who see residents and attendings in the above-described ambulatory setting and privately insured women who only see attending providers in outpatient practices. For this subset, the electronic medical record was further queried to determine attendance at a postpartum visit, contraceptive counseling at a postpartum visit, and contraceptive use at the time of a postpartum visit, with a postpartum visit defined as an outpatient visit within 12 weeks of the delivery date.

Women were identified as using a tier 1 or tier 2 contraceptive method based on the World Health Organization (WHO) classification system. ${ }^{21}$ Tier 1 methods include any IUD, any subdermal implant, or female or male sterilization. Tier 2 methods include any oral contraceptive pill, depomedroxyprogesterone acetate (DMPA), transdermal patch, vaginal ring, and lactational amenorrhea. Other tiers include male condoms, female condoms, diaphragm, sponge, or fertility awareness-based methods (tier 3) or withdrawal or spermicide (tier 4).

Patients whose male partners planned a vasectomy, but who had not yet received it, were coded as using no method unless they also chose an additional method, as were women who reported that their partners carried a diagnosis of azoospermia and women who reported abstinence only. Patients who underwent peripartum hysterectomy were considered tier 1 for the purposes of this analysis, as were women who reported only female sexual partners.

Women were coded as using a method if they underwent sterilization, received a DMPA injection, or actually received a prescription for an oral contraceptive pill, transdermal patch, or vaginal ring, not merely if they reported a plan for use. Of note, inpatient placement of IUDs and subdermal implants was not available during the time period of this study, so patients were only counted as receiving tier 1 or tier 2 contraception before hospital discharge if they reported a plan for IUD or implant use and actually received it at their postpartum visit. Women could change methods between hospital discharge and the postpartum period; two separate variables were used for these separate time periods. Women who reported dual contraceptive use (such as condoms and DMPA) were classified according to the highest tier possible given the multiple methods reported.

Maternal demographic and clinical characteristics that correlated with postpartum contraceptive counseling and use were abstracted from the medical record. Variables include maternal race and ethnicity (non-Hispanic white, non-Hispanic black, Hispanic, Asian, or other), maternal age (years), parity, insurance payer status (public or private; in Illinois, all pregnant women are automatically granted Medicaid if they do not otherwise have insurance, regardless of citizenship, immigration status, or economic status, thus there are no truly uninsured patients in this sample), gestational age at delivery (weeks), twin gestation, and mode of delivery (spontaneous vaginal delivery, operative vaginal delivery, or cesarean delivery). Race and ethnicity are self-reported on admission; women can choose "other race" or decline to answer.

Student's $t$-test or chi-square analyses were used for continuous and categorical variables, respectively, in bivariable analyses. Multivariable logistic regression was used to identify 
factors independently associated with inpatient contraception counseling as well as tier 1 or tier 2 contraceptive method uptake in both inpatient and outpatient settings. Multivariable models included all potential confounders listed in the above paragraph. All hypothesis tests were two-tailed; variables were considered significant at the $p<0.05$ level. All analyses were carried out in STATA (version 14.2; StataCorp, College Station, TX). Approval for this study was obtained from the Northwestern Institutional Review Board with a waiver of informed consent.

\section{Results}

Of 594 eligible women, $40.9 \%$ were non-Hispanic white, $25.3 \%$ were non-Hispanic black, $12.3 \%$ were Hispanic, $4.7 \%$ were Asian, and the remaining $16.2 \%$ were of other or unknown race/ethnicity. One-third $(33.7 \%)$ received publicly insured prenatal care. The mean gestational age at delivery was $29.3 \pm 2.3$ weeks (Table 1). In this cohort, $34.8 \%(n=207)$ had available outpatient records for review. Of this subgroup, $84.5 \%(n=175)$ attended a postpartum visit (Fig. 1).

Non-Hispanic black women were more likely to have outpatient records available (46.1\% had records, compared with $31.7 \%$ of non-Hispanic whites, $23.3 \%$ of Hispanics, $42.3 \%$ of Asians, and $31.3 \%$ of women of other race, $p=0.004)$. The women with outpatient records available were more likely to have received inpatient counseling $(38.5 \%$ vs. $56.0 \%, p<0.001$ ), but no more likely to adopt a tier 1 or tier 2 method before leaving the hospital (19.9\% vs. $24.2 \%$, $p=0.22$ ). There were otherwise no significant differences between women who had outpatient records available compared with those who did not. Of women with postpartum records available, women who were older $(32.2 \pm 6.0$ vs. $29.0 \pm 7.9$ years, $p=0.01)$ and had private insurance $(88.9 \%$ of those with private insurance vs. $76.4 \%$ of those with public insurance, $p=0.02$ ) were more likely to attend their postpartum visit.

Of the full cohort, $44.6 \%(n=265)$ had documented inpatient contraceptive counseling, but only $21.4 \%(n=127)$ left

Table 1. Characteristics of a Cohort of Women With a Recent Preterm Birth Between 23 and 32 Weeks' Gestational Age

\begin{tabular}{lc}
\hline Maternal characteristic & $\mathrm{n}(\%)$ or mean $\pm S D$ \\
$(\mathrm{n}=594)$
\end{tabular}

${ }^{\text {a }}$ Parity refers to parity before the delivery of the current pregnancy. $\mathrm{SD}$, standard deviation. the hospital using or planning for a WHO tier 1 or tier 2 method (Fig. 1). Of these 127 women, 57.6\% $(n=72)$ received DMPA; $21.3 \%(n=27)$ underwent postpartum sterilization or had solely a female partner $(n=4) ; 12.6 \%(n=16)$ planned for (and received) an IUD at their postpartum visit; and $9.5 \%$ $(n=12)$ received a prescription for combined hormonal contraception.

Women who were younger, non-Hispanic black, multiparous, and delivered at earlier gestational ages were more likely to have counseling documented (Table 2). Notably, compared with women with private insurance, women with public insurance were significantly more likely to have documented counseling ( $22.8 \%$ of privately insured patients vs. $87.5 \%$ of publicly insured patients, $p<0.001)$. This finding persisted when accounting for confounding factors (adjusted odds ratio [aOR] 9.55, 95\% confidence interval [CI] 5.31-17.2).

Additionally, women with public insurance were also more likely to be discharged from the hospital postpartum with uptake of a WHO tier 1 or 2 method as an inpatient (5.8\% of privately insured patients vs. $52.0 \%$ of publicly insured patients, $p<0.001$ ), a finding that also persisted after adjusting for confounding factors (aOR 9.51, 95\% CI 4.7818.9). Non-Hispanic black and Asian women also were more likely to be discharged with a WHO tier 1 or 2 method.

Of the 175 women for whom outpatient records were available and who attended a postpartum visit, $53.7 \%(n=94)$ of women received inpatient contraceptive counseling and 98.3\% $(n=172)$ of attendees received contraceptive counseling at the postpartum visit. However, in this subgroup, only $22.3 \% \quad(n=39)$ of the 175 women who attended a postpartum visit adopted a WHO tier 1 or tier 2 method before hospital discharge and only $54.9 \%(n=96)$ adopted a tier 1 or tier 2 method by the completion of the postpartum visit (Fig. 1). Public insurance and multiparity were positively associated with the use of a WHO tier 1 or 2 method, while twin gestation was negatively associated with uptake of a WHO tier 1 or 2 method (Table 3 ).

Of the 32 women who had medical records available, but did not attend a postpartum visit, only $34.4 \%(n=11)$ had received tier 1 or tier 2 contraception before hospital discharge. The majority of these women $(6 / 11,54.6 \%)$ received DMPA. Although a small sample, those who had received DMPA as an inpatient were no more or less likely to present for their postpartum visit $(p=0.30)$.

\section{Discussion}

Rapid repeat pregnancies (within 18 months of a previous birth) are common in the United States. ${ }^{11}$ The proportion of rapid repeat pregnancies is likely higher among women following a preterm birth as they are less likely to use effective contraception, $^{22}$ yet these women are at highest risk of a recurrent preterm birth. ${ }^{7}$ Counseling regarding the role of contraception in lengthening the interpregnancy interval could improve outcomes in subsequent pregnancies.

Our data demonstrate, however, that this counseling following a preterm birth is far from universal, with the majority of women failing to receive documented inpatient contraception counseling. Moreover, only a small majority of women had documented uptake of a WHO tier 1 or 2 method by 12 weeks' postpartum, a clear risk factor for a shortinterval pregnancy. ${ }^{23}$ Importantly, substantial differences in 
Inpatient sample:

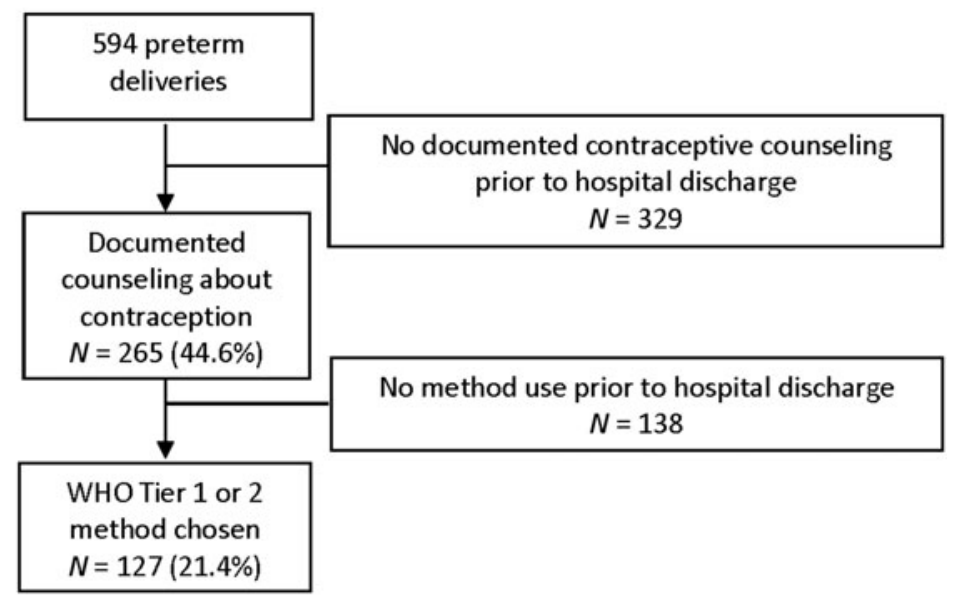

Outpatient sample:

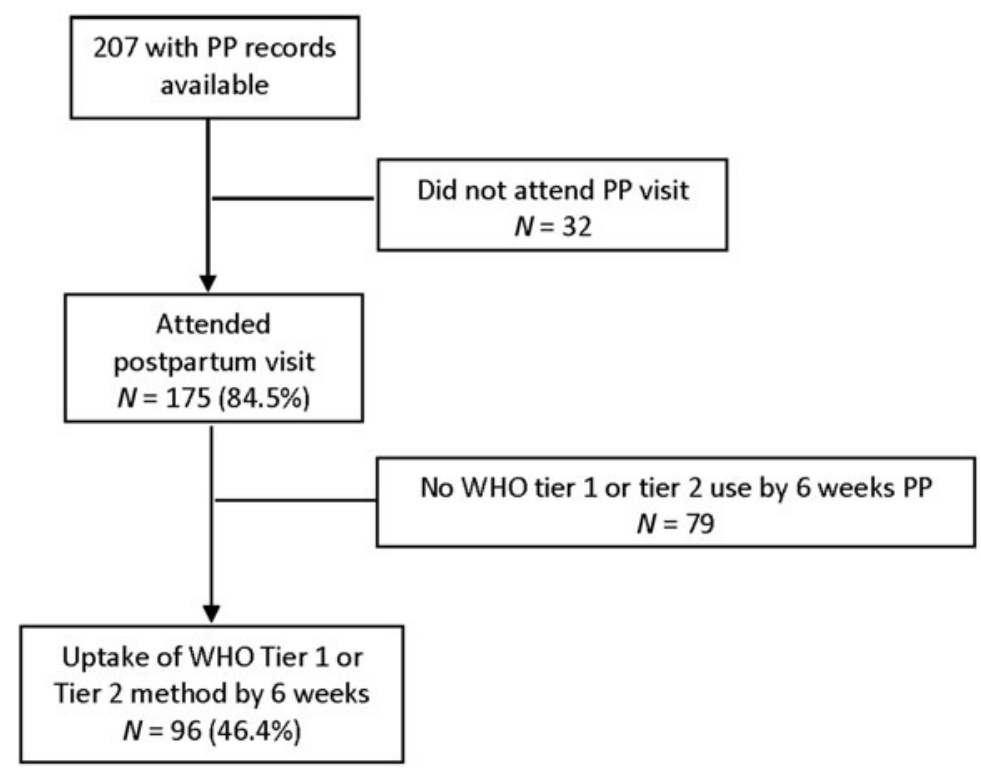

FIG. 1. Composition of inpatient and outpatient study samples. both counseling and uptake exist based on insurance status, race, age, and parity.

In our sample, there was a low prevalence of inpatient contraceptive counseling. The reasons for this low rate are unclear, but previous research indicates that providers are hesitant to address contraception with patients following a preterm delivery, especially if their infants are at risk of neonatal demise. ${ }^{22}$ Alternatively, providers may think of contraceptive counseling as an item that can wait until the postpartum visit.

Although contraceptive counseling at postpartum visits was nearly universal and the postpartum attendance rate in this cohort was higher than in other reports, a substantial proportion of women did not attend a postpartum visit. Attendance at a postpartum visit has been shown to lengthen interpregnancy intervals via increased use of highly effective contraception. $^{23,24}$ Previous studies have shown poor postpartum attendance rates, ${ }^{25}$ indicate that patients prefer to be counseled at several points pre- and postdelivery, ${ }^{26}$ and show that inpatient postpartum provision of long-acting reversible contraception (LARC) can increase uptake, decrease discontinuation, and decrease unintended pregnancy rates. ${ }^{27-29}$ These existing reports in concert with our data on incomplete postpartum return to care indicate that increasing inpatient (intrapartum or early postpartum) contraceptive counseling and LARC provision (a service not routinely available at our hospital during the study period) following a preterm delivery represents an area of potential improvement, particularly since women delivering preterm may not have had sufficient outpatient antenatal contraceptive counseling before delivery.

Our findings show that contraceptive counseling focuses on women with multiple risk factors for preterm birth: nonHispanic black race/ethnicity, public insurance, and younger age. Yet, all the women in our study share the most important risk factor: a prior preterm birth. ${ }^{30}$ Women with private insurance in our study were undercounseled. Perhaps providers 


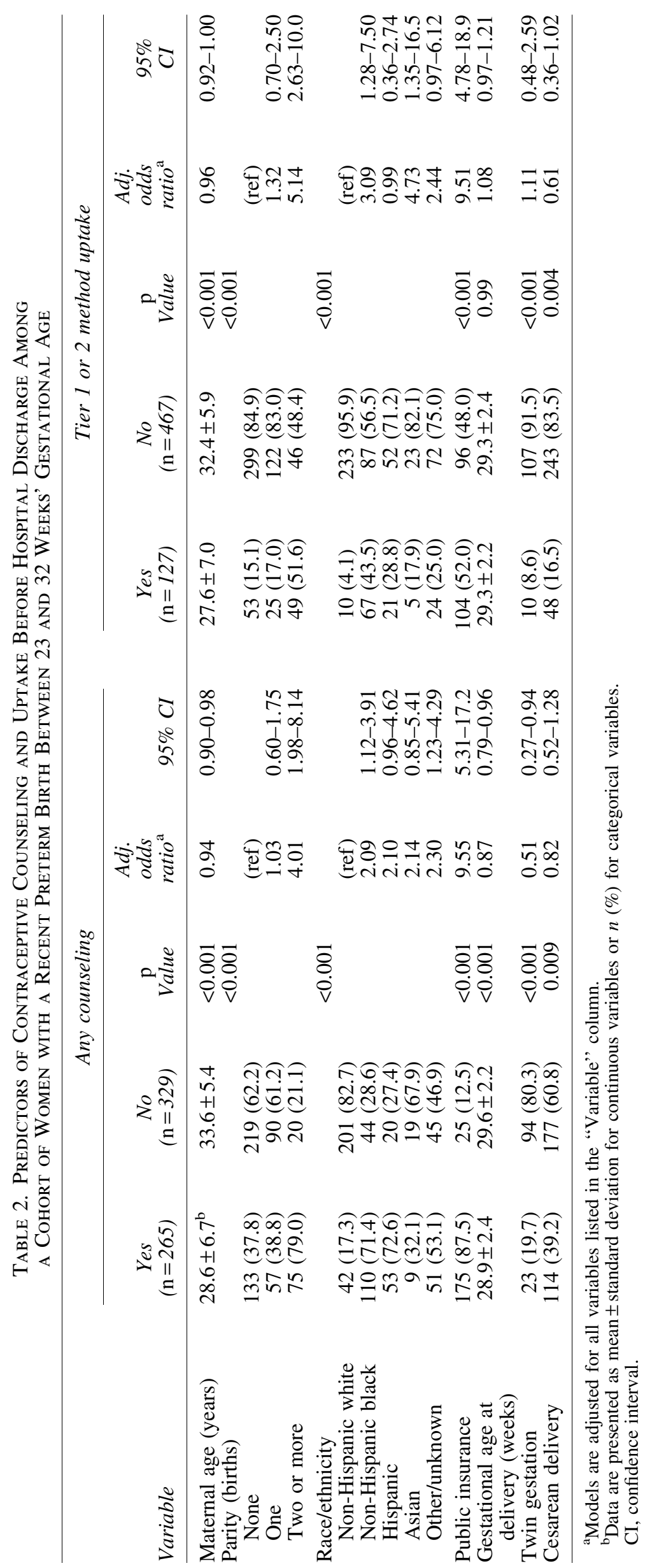


Table 3. Predictors of Contraceptive Use at Postpartum Visits Among a Cohort of Women with a Recent Preterm Birth Between 23 and 32 Weeks' Gestational Age

\begin{tabular}{|c|c|c|c|c|c|}
\hline \multirow[b]{2}{*}{ Variable } & \multicolumn{5}{|c|}{ Tier one or tier two method use } \\
\hline & Yes $(\mathrm{n}=96)$ & No $(\mathrm{n}=79)$ & p Value & Adj. odds ratio $\mathbf{a}^{\mathbf{a}}$ & $95 \% C I$ \\
\hline Maternal age (years) & $30.9 \pm 6.1^{b}$ & $33.8 \pm 5.5$ & 0.001 & 0.93 & $0.87-1.00$ \\
\hline Parity (births) & & & 0.004 & & \\
\hline None & $46(44.7)$ & $57(55.3)$ & & (ref) & \\
\hline One & $29(65.9)$ & $15(34.1)$ & & 2.39 & $1.00-5.71$ \\
\hline Two or more & $21(75.0)$ & $7(25.0)$ & & 3.40 & $1.05-11.0$ \\
\hline Race/ethnicity & & & 0.013 & & \\
\hline Non-Hispanic white & $29(40.9)$ & $42(59.1)$ & & (ref) & \\
\hline Non-Hispanic black & 39 (69.6) & $17(30.4)$ & & 0.79 & $0.27-2.26$ \\
\hline Hispanic & $9(75.0)$ & $3(25.0)$ & & 0.62 & $0.11-3.34$ \\
\hline Asian & $6(54.6)$ & $5(45.5)$ & & 1.45 & $0.34-6.09$ \\
\hline Other/unknown & $13(52.0)$ & $12(48.0)$ & & 1.59 & $0.53-4.70$ \\
\hline Public insurance & $45(81.8)$ & $10(18.2)$ & $<0.001$ & 4.84 & $1.74-13.5$ \\
\hline Gestational age at delivery (weeks) & $29.4 \pm 2.4$ & $29.1 \pm 2.2$ & 0.32 & 1.11 & $0.94-1.30$ \\
\hline Twin gestation & $5(16.7)$ & $25(83.3)$ & $<0.001$ & 0.16 & $0.05-0.52$ \\
\hline Cesarean delivery & $48(52.2)$ & $44(47.8)$ & 0.45 & 0.81 & $0.40-1.66$ \\
\hline
\end{tabular}

a Models are adjusted for all variables listed in the "Variable" column.

${ }^{\mathrm{b}}$ Data are presented as mean \pm standard deviation for continuous variables or $n(\%)$ for categorical variables.

of privately insured patients assume that these patients will ask for contraception on their own and do not perceive them to be at risk of recurrent preterm birth. Private providers may also prefer to counsel women in the outpatient setting as they believe their patients will return for care and will not have resumed sexual activity before outpatient care. However, relying on postpartum outpatient counseling would leave women at risk of being missed for contraceptive counseling as the attendance at postpartum visits is never universal, ${ }^{31}$ including in this sample.

Alternately, at our institution, most publicly insured patients are seen by both resident and attending physicians, whereas resident and attending physicians both see only privately insured patients who have had medically complicated deliveries. Whether having residents involved in postpartum care truly improves contraceptive counseling or merely improves documentation of this counseling is unknown. Furthermore, providers may have focused inpatient counseling on publicly insured patients as they may have been more concerned about these women's access to insurance lapsing shortly following delivery.

This study has several strengths, including a large diverse population of women who experienced a preterm birth. Second, the ability to search the medical record for documented counseling, however briefly noted, as well as contraceptive uptake allows us to examine contraceptive decisions on a level that using administrative data does not.

This study also has several limitations. First, all data are retrospective and thus reflect documented counseling and care, which may differ from what women actually received. It is possible that some providers counseled about contraception, but failed to document such counseling; this failure of documentation is also likely nonrandom as attending physician documentation was noticeably scanter than resident documentation. However, we assessed contraception uptake via multiple channels (including discharge instructions, medical administration records, and prescriptions) and thus did not rely on provider notes alone. Second, outpatient re- cords were only available for a portion of the overall sample. Women who did not have outpatient records were overwhelmingly receiving care in private practices affiliated with this hospital; private practitioners may intentionally counsel patients exclusively in the outpatient setting. Third, women may have chosen to use contraceptive methods such as condoms without any discussion with providers, received contraception from another source, or received a method following their postpartum visit, although we suspect this was uncommon given that the majority of women returned to their primary provider for postpartum care.

Fourth, these results derive from a single tertiary care institution and may not be applicable in other settings. Specifically, we hypothesize that the higher frequency of contraception counseling documentation for publicly insured patients may be partially related to resident involvement, and as such, these data may be less generalizable to settings without resident presence. Fifth, women who experienced lengthy antepartum stays may have been more likely to be counseled due both to the time they spent in the hospital and because all antepartum patients receive care from residents in our hospital.

Sixth, we make no attempt to determine the quality of contraceptive counseling, only whether any discussion occurred. Providers may have mentioned only one or a few methods, thus not truly counseling women regarding their full range of options. Finally, all data are correlational only; causality cannot be implied. Furthermore, even if contraceptive counseling and uptake were improved, this does not imply that preterm birth rates would necessarily fall: as Klebanoff ${ }^{12}$ noted in a recent editorial, it is unclear whether the interpregnancy interval is causal or merely a marker of other risk factors for preterm birth.

In this cohort of women at high risk for recurrent preterm birth, women at the highest risk of recurrent preterm birth were the most likely to receive counseling and effective contraception. While we are reassured that many at-risk women received contraceptive care, the dramatic disparities 
by race/ethnicity and payer status raise substantial concern regarding perhaps overemphasis on contraception for sociodemographically vulnerable and historically stigmatized groups and underemphasis for others. Indeed, previous research suggests that women who are overcounseled regarding contraception can even be counterproductive if women feel coerced or singled out for attention based on racial or socioeconomic status. ${ }^{32}$ Improved universal inpatient and outpatient counseling could increase the use of WHO tier 1 or 2 contraceptive methods, decrease the interpregnancy interval, and, critically, decrease both the risk of recurrent preterm birth and disparities in preterm birth.

\section{Acknowledgments}

L.M.Y. is supported by the NICHD K12 HD050121-11. Research reported in this publication was supported, in part, by the National Institutes of Health's National Center for Advancing Translational Sciences, Grant Number UL1TR001422. The content is solely the responsibility of the authors and does not necessarily represent the official views of the National Institutes of Health.

\section{Author Disclosure Statement}

No competing financial interests exist.

\section{References}

1. Martin JA, Hamilton BE, Osterman MJ, Driscoll AK, Mathews TJ. Births in the United States, 2015. Hyattsville, MD: National Center for Health Statistics, 2016.

2. March of Dimes, World Health Organization. Born too soon: The global action report on pre-term birth. Geneva, Switzerland: WHO, 2012.

3. Mwaniki MK, Atieno M, Lawn JE, Newton CR. Longterm neurodevelopmental outcomes after intrauterine and neonatal insults: A systematic review. Lancet 2012;379: 445-452.

4. Lawn JE Blencowe $\mathrm{H}$, Oza S, et al. Every newborn: Progress, priorities, and potential beyond survival. Lancet 2014;384:189-205.

5. MacDorman MF, Mathews TJ. Understanding racial and ethnic disparities in U.S. infant mortality rates. NCHS Data Brief 2011:1-8.

6. Office of Disease Prevention and Health Promotion Healthy People 2020 Topics and Objectives. Washington, DC: USDHHS. Available at: https://healthypeople.gov/2020/topicsobjectives/topic/maternal-infant-and-child-health/objectives Accessed February 18, 2017.

7. Spong CY. Prediction and prevention of recurrent spontaneous preterm birth. Obstet Gynecol 2007;110:405-415.

8. Conde-Agudelo A, Rosas-Bermudez A, Kafury-Goeta AC. Birth spacing and risk of adverse perinatal outcomes, a meta-analysis. JAMA 2006;295:1809-1823.

9. Wendt A, Gibbs CM, Peters S, Hogue CJ. Impact of increasing inter-pregnancy interval on maternal and infant health. Paediatr Perinat Epidemiol 2012;26 Suppl 1:239 258 .

10. DeFranco EA, Stamilio DM, Boslaugh SE, Gross GA, Muglia LJ. A short interpregnancy interval is a risk factor for preterm birth and its recurrence. Am J Obstet Gynecol 2007;197:264.e1-264.e6.
11. Gemmill A, Lindberg LD. Short interpregnancy intervals in the United States. Obstet Gynecol 2013;122:64-71.

12. Klebanoff MA. Interpregnancy interval and pregnancy outcomes: Causal or not? Obstet Gynecol 2017;129:405-407.

13. Hanley GE, Hutcheon JA, Kinniburgh BA, Lee L. Interpregnancy interval and adverse pregnancy outcomes: An analysis of successive pregnancies. Obstet Gynecol 2017; 129:408-415.

14. Khoshnood B, Lee KS, Wall S, Hsieh HL, Mittendorf R. Short interpregnancy intervals and the risk of adverse birth outcomes among five racial/ethnic groups in the United States. Am J Epidemiol 1998;148:798-805.

15. Isquick $\mathrm{S}$, Chang $\mathrm{R}$, Thiel de Bocanegra $\mathrm{H}$, Chabot $\mathrm{M}$, Brindis CD. Postpartum contraception and interpregnancy intervals among adolescent mothers accessing public services in California. Matern Child Health J 2017;21: 752-759.

16. Thiel de Bocanegra H, Braughton M, Bradsberry M, Howell M, Logan J, Schwarz EB. Racial and ethnic disparities in postpartum care and contraception in California's Medicaid program. Am J Obstet Gynecol 2017;217:47.e147.e7.

17. Brunson MR, Klein DA, Olsen CH, Weir LF, Roberts TA. Postpartum contraception: Initiation and effectiveness in a large universal healthcare system. Am J Obstet Gynecol 2017;217:55.e1-55.e9.

18. Rodriguez MI, Chang R, Thiel de Bocanegra H. The impact of postpartum contraception on reducing preterm birth: Findings from California. Am J Obstet Gynecol 2015;213: 703 e701-e706.

19. Zapata LB MS, Whiteman MK, Jamieson DJ, et al. Contraceptive counseling and postpartum contraceptive use. Am J Obstet Gynecol 2015;212:171e.171-178.

20. Schaaf JM, Hof MH, Mol BW, Abu-Hanna A, Ravelli AC. Recurrence risk of preterm birth in subsequent singleton pregnancy after preterm twin delivery. Am J Obstet Gynecol 2012;207:279 e271-e277.

21. World Health Organization. Comparing typical effectiveness of contraceptive methods, 2006. Available at: www .fhi.org/nr/shared/enFHT/Resources/EffectivenessChart.pdf Accessed February 18, 2017.

22. Robbins CL, Farr SL, Zapata LB, D'Angelo DV, Callaghan WM. Postpartum contraceptive use among women with a recent preterm birth. Am J Obstet Gynecol 2015;213: 508.e1-508.e9.

23. Thiel de Bocanegra H CR, Menz M, Howell M, Darney P. Postpartum contraception in publicly-funded programs and interpregnancy intervals. Obstet Gynecol 2013;122(2 Pt 1):296-303.

24. Harney C, Dude A, Haider S. Factors associated with short interpregnancy interval in women who plan postpartum LARC: A retrospective study. Contraception 2017;95:245-250.

25. American College of Obstetricians and Gynecologists' Committee on Obstetric Practice, Association of Women's Health Obstetric and Neonatal Nurses. Committee Opinion No. 666: Optimizing postpartum care. Obstet Gynecol 2016;127:e187-e192.

26. Yee L, Simon M. Urban minority women's perceptions of and preferences for postpartum contraceptive counseling. J Midwifery Womens Health 2011;56:54-60.

27. Rodriguez MI, Evans M, Espey E. Advocating for immediate postpartum LARC: Increasing access, improving outcomes, and decreasing cost. Contraception 2014;90: $468-471$. 
28. Crockett AH, Pickell LB, Heberlein EC, Billings DL, Mills B. Six- and twelve-month documented removal rates among women electing postpartum inpatient compared to delayed or interval contraceptive implant insertions after Medicaid payment reform. Contraception 2017;95: 71-76.

29. Tocce KM, Sheeder JL, Teal SB. Rapid repeat pregnancy in adolescents: Do immediate postpartum contraceptive implants make a difference? Am J Obstet Gynecol 2012;206: 481.e1-481.e7.

30. Yang J, Baer RJ, Berghella V, et al. Recurrence of preterm birth and early term birth. Obstet Gynecol 2016;128: 364-372.

31. Wilcox A, Levi EE, Garrett JM. Predictors of non-attendance to the postpartum follow-up visit. Matern Child Health J 2016;20(Suppl 1):22-27.
32. Yee LM, Simon MA. Perceptions of coercion, discrimination and other negative experiences in postpartum contraceptive counseling for low-income minority women. J Health Care Poor Underserved 2011;22:1387-1400.

Address correspondence to: Annie Dude, $M D, P h D$ Division of Maternal-Fetal Medicine Department of Obstetrics and Gynecology

Feinberg School of Medicine Northwestern University 250 East Superior Street Suite 5-2185 Chicago, IL 60611

E-mail: ann.dude@northwestern.edu 